\title{
Skewed Reflected Distributions Generated by the Laplace Kernel
}

\author{
M. Masoom $\mathrm{Ali}^{1}$, Manisha $\mathrm{Pal}^{2}$ and Jungsoo $\mathrm{Woo}^{3}$ \\ ${ }^{1}$ Dept. of Mathematical Sciences, Ball State University, Indiana, USA \\ ${ }^{2}$ Dept. of Statistics, University of Calcutta, India \\ ${ }^{3}$ Dept. of Statistics, Yeungnam University, Gyongsan, South Korea
}

\begin{abstract}
In this paper we construct some skewed distributions with pdfs of the form $2 f(u) G(\lambda u)$, where $\lambda$ is a real number, $f(\cdot)$ is taken to be a Laplace pdf while the cdf $G(\cdot)$ comes from one of Laplace, double Weibull, reflected Pareto, reflected beta prime, or reflected generalized uniform distribution. Properties of the resulting distributions are studied. In particular, expressions for the moments of these distributions and the characteristic functions are derived. However, as some of these quantities could not be evaluated in closed forms, special functions have been used to express them. Graphical illustrations of the pdfs of the skewed distributions are also given. Further, skewness-kurtosis graphs for these distributions have been drawn.
\end{abstract}

Zusammenfassung: In diesem Aufsatz konstruieren wir einige schiefe Verteilungen mit Verteilungsfunktionen der Form $2 f(u) G(\lambda u)$, wobei $\lambda$ eine reelle Zahl und $f(\cdot)$ eine Laplace Dichte ist, während die Verteilungsfunktion $G(\cdot)$ von einer Laplace-, zweiseitigen Weibull-, reflektierten Pareto-, reflektierten Beta-Primär-, oder reflektierten generalisierten Gleichverteilung stammt. Die Eigenschaften der resultierenden Verteilungen werden untersucht. Im Besonderen werden Ausdrücke für die Momente dieser Verteilungen und deren charakteristische Funktionen hergeleitet. Da jedoch einige dieser Größen nicht in geschlossener Form dargestellt werden konnten, wurden spezielle Funktionen verwendet um diese auszudrücken. Graphische Illustrationen der Dichten dieser schiefen Verteilungen sind auch gegeben. Weiters sind Skewness-Kurtosis Graphen für diese Verteilungen gezeichnet worden.

Keywords: Skewed Distribution, Laplace Distribution, Double Weibull Distribution, Reflected Pareto Distribution, Reflected Beta Prime Distribution, Reflected Generalized Uniform Distribution, Moments, Characteristic Functions.

\section{Introduction}

Univariate skew-symmetric models have been defined by several authors, using the concept described in the article by Azzalini (1985). This particular article defined the family of univariate skewed-normal distributions, an extension of the symmetric normal distribution to a general class of asymmetrical distributions. An extension of the skewed-normal distribution to the multivariate case was studied by Azzalini and Dalla Valle (1996). Its application in statistics has been considered by Azzalini and Capitanio (1999). As a general result, Azzalini (1985) showed that any symmetric distribution could be viewed as 
a member of more general class of skewed distributions. In recent years, many authors have studied similar distributions. For example, see Ali, Woo, Pal, and Wahed (2008), Ali and Woo (2006), Gupta, Chang, and Huang (2002), Wahed and Ali (2001), Arnold and Beaver (2000a, 2000b), Balakrishnan and Ambagaspitiy (1994), Azzalini (1986). The main feature of these models is that a skewness parameter $\lambda$ is introduced to control skewness and kurtosis. These models are useful in many practical situations (see Hill and Dixon, 1982; Arnold, Beaver, Groeneveld, and Meeker, 1983) and have also been used in studying robustness and as priors in Bayesian estimation (see O'Hagan and Leonard, 1976; Mukhopadhyay and Vidakovic, 1995).

The skew-symmetric models defined by different researchers are based on the following general result by Azzalini (1985):

Lemma: Let $U$ and $V$ be two arbitrary absolutely continuous independent random variables symmetric about zero, with probability density functions (pdfs) $f$ and $g$, and cumulative distribution functions (cdfs) $F$ and $G$, respectively. Then for any $\lambda \in \mathbb{R}$, the function

$$
f_{Z}(z ; \lambda)=2 f(z) G(\lambda z)
$$

is a valid pdf of a random variable, say $Z$.

The papers mentioned above obtain skew-symmetric models by taking both $f$ and $G$ to belong to the same family of symmetric distributions. Mukhopadhyay and Vidakovic (1995) pointed out an extension of the above approach by suggesting that one take $f$ and $G$ in (1) to belong to two different families. Nadarajah and Kotz (2003) were probably the first to consider this extension. They studied skew-symmetric models with $f$ as the pdf of a normal distribution with mean zero and variance $\sigma^{2}$, and $G$ as the cdf of normal, Student's $t$, Cauchy, Laplace, logistic and uniform distributions. Nadarajah and Kotz (2004) also defined skew-symmetric models with $f$ as the pdf of a Laplace distribution, and $G$ being the cdf of normal, Student's $t$, Cauchy, logistic and uniform distributions. Ali, Pal, and Woo (2008) discussed skew-symmetric models with reflected gamma kernel and $G$ as the cdf of Laplace, double Weibull, reflected Pareto, reflected beta prime and reflected generalized uniform distributions.

In this paper we construct skewed distributions with a Laplace kernel. Here $f$ is given by

$$
f(x)=\frac{1}{2} \theta \exp (-|x| / \theta), \quad x \in \mathbb{R}, \quad \theta>0 .
$$

We then explicitly define skewed Laplace-Laplace, Laplace-double Weibull, Laplacereflected Pareto, Laplace-reflected beta prime and skewed Laplace-reflected generalized uniform distributions. These distributions reduce to the symmetric Laplace distribution when the skewing parameter is set to zero. They allow distinct local maxima for bimodal distributions and therefore provide greater flexibility for modeling bimodal frequency distributions. We also derive the expressions for the pdf, cdf, and the moments of these distributions. However, some of these quantities could not be evaluated in closed forms and therefore special functions were used to express them. Also, we find moments of the distributions only for the skewness parameter $\lambda>0$ because of the following:

Fact 1: For a random variable $Z$ having pdf given by (1),

$$
\mathrm{E}\left(Z^{k} ; \lambda\right)=(-1)^{k} \mathrm{E}\left((-Z)^{k} ;-\lambda\right) .
$$


The paper is organized as follows. In Sections 2 to 7 we introduce the skewed distributions and derive some of their properties. In Section 8, we discuss the skewness and kurtosis of these distributions. In Section 9 we conclude the article with some remarks on our findings.

\section{Skew Laplace-Laplace Model}

The cdf of a Laplace variable is given by

$$
G(x)=\frac{1}{2}[1+\operatorname{sgn}(x)(1-\exp (-|x| / \beta))], \quad x \in \mathbb{R}, \quad \beta>0,
$$

where

$$
\operatorname{sgn}(x)=\left\{\begin{aligned}
1 & \text { if } x \geq 0 \\
-1 & \text { if } x<0
\end{aligned}\right.
$$

Then (2) yields the following pdf of the skew Laplace-Laplace variable $Z$ :

$$
f_{Z}(z ; \lambda)=\frac{1}{2 \theta} \exp (-|z| / \theta)[1+\operatorname{sgn}(\lambda z)(1-\exp (-|\lambda z| / \beta))], \quad z \in \mathbb{R}, \quad \lambda \in \mathbb{R} .
$$

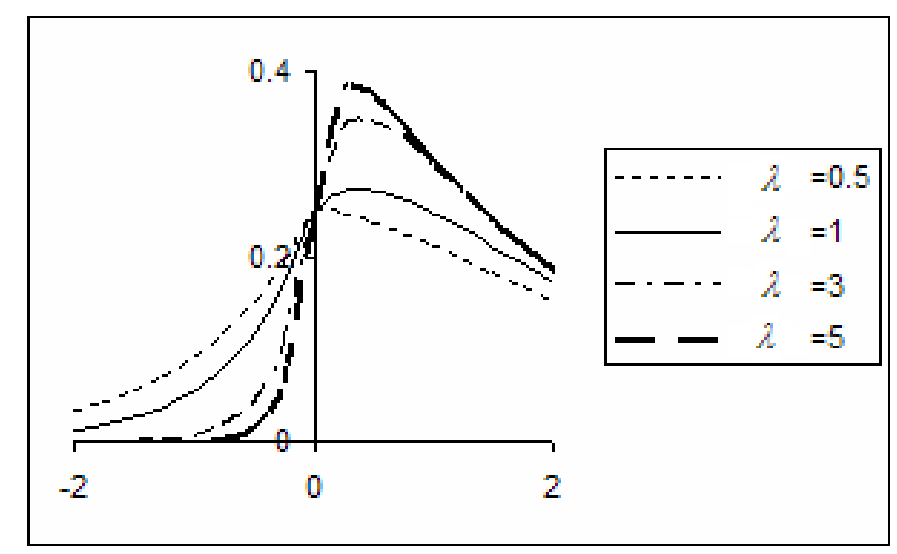

Figure 1: Density curves of skew Laplace-Laplace distributions for $\lambda=0.5,1,3,5$ when $\theta=2, \beta=1$.

The $k$-th moment of the distribution is obtained as

$$
\mathrm{E}\left(Z^{k} ; \lambda\right)=\theta^{k} \Gamma(k+1)\left[1+\frac{(-1)^{k}-1}{2} \frac{\beta^{k+1}}{(\beta+\lambda \theta)^{k+1}}\right], \quad \lambda>0 .
$$

Measures of skewness and kurtosis of the distribution are, respectively, given by

$$
\gamma_{1}=\frac{\mu_{3}}{\mu_{2}^{3 / 2}}, \quad \gamma_{2}=\frac{\mu_{4}}{\mu_{2}^{2}}
$$

where $\mu_{k}$ denotes $k$-th order central moment of $Z$, with

$$
\begin{aligned}
& \mu_{2}=\theta^{2}\left(1-2 \rho^{2}-\rho^{4}\right), \quad \mu_{3}=2 \theta^{3}\left(1-\rho^{6}\right), \\
& \mu_{4}=3 \theta^{4}\left(3-8 \rho^{2}+2 \rho^{4}-8 \rho^{6}-\rho^{8}\right), \quad \rho=\frac{\beta}{\beta+\lambda \theta} .
\end{aligned}
$$


For $\theta=\beta$, we get

$$
\mathrm{E}\left(Z^{k} ; \lambda\right)=\theta^{k} \Gamma(k+1)\left[1+\frac{(-1)^{k}-1}{2} \frac{1}{(1+\lambda)^{k+1}}\right] .
$$

Then, the first four moments of the distribution come out to be

$$
\begin{aligned}
& \mathrm{E}(Z ; \lambda)=\theta^{k} \Gamma(k+1)\left[1-\frac{1}{(1+\lambda)^{2}}\right], \quad \mathrm{E}\left(Z^{2} ; \lambda\right)=2 \theta^{2} \\
& \mathrm{E}\left(Z^{3} ; \lambda\right)=6 \theta^{k}\left[1-\frac{1}{(1+\lambda)^{4}}\right], \quad \mathrm{E}\left(Z^{4} ; \lambda\right)=24 \theta^{4} .
\end{aligned}
$$

Measure of skewness of the distribution is then given by

$$
\gamma_{1}=\frac{2\left[1-\left(\frac{1}{1+\lambda}\right)^{6}\right]}{\left[1+2\left(\frac{1}{1+\lambda}\right)^{2}-\left(\frac{1}{1+\lambda}\right)^{4}\right]^{3 / 2}} .
$$

Figure 2 shows that the distribution is negatively skewed and the skewness increases as $\lambda$ increases.

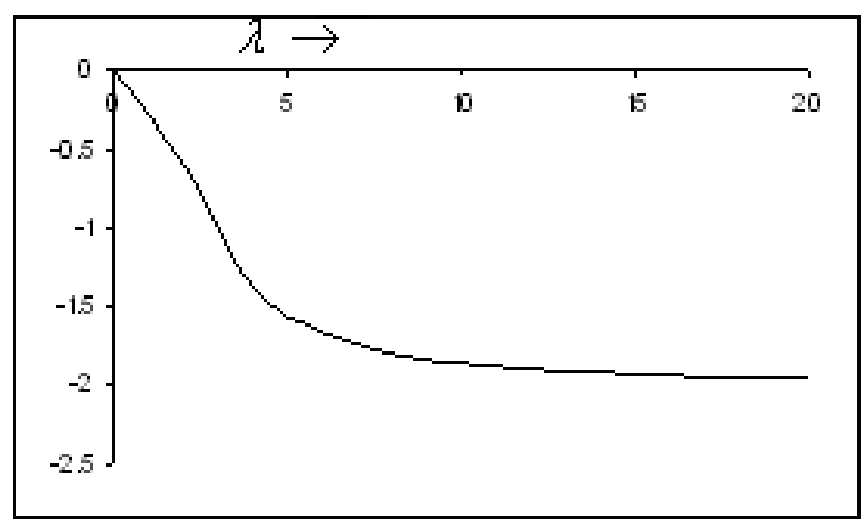

Figure 2: Graph of $\gamma_{1}$ versus $\lambda$ when $\theta=\beta$.

The characteristic function of the skew Laplace-Laplace distribution defined by (3) has the form

$$
\phi_{Z}(t)=(1-i t \theta)^{-1}+\frac{1}{2 \theta}\left[\left(\frac{1}{\theta}+\frac{\lambda}{\beta}+i t\right)^{-1}-\left(\frac{1}{\theta}+\frac{\lambda}{\beta}-i t\right)^{-1}\right]
$$

\section{Skew Laplace-Double Weibull Model}

The double Weibull random variable has the cdf

$$
G(x)=\frac{1}{2}\left[1+\operatorname{sgn}(x)\left(1-\exp \left(-|x|^{\alpha}\right)\right)\right], \quad x \in \mathbb{R}, \quad \alpha>0 .
$$




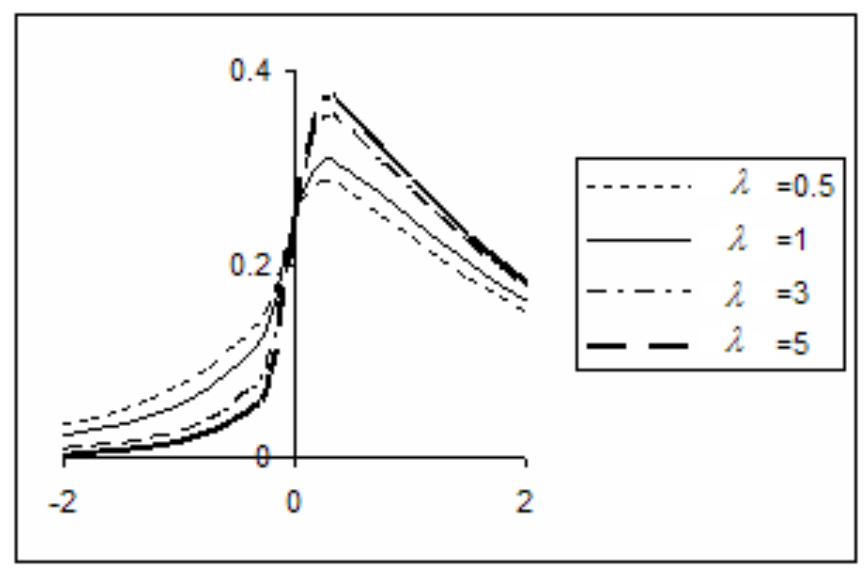

Figure 3: Density curves of skew Laplace-double Weibull distributions for $\lambda=0.5,1,3,5$ when $\theta=2, \alpha=0.5$.

The skew Laplace-double Weibull distribution is, therefore, defined by the pdf $f_{Z}(z ; \lambda)=\frac{1}{2 \theta} \exp (-|z| / \theta)\left[1+\operatorname{sgn}(\lambda z)\left(1-\exp \left(-|\lambda z|^{\alpha}\right)\right)\right], \quad z, \lambda \in \mathbb{R}, \alpha, \theta>0$.

From formula 3.381(4) in Gradshteyn and Ryzhik (1965) we obtain the $k$-th moment of the distribution as

$$
\mathrm{E}\left(Z^{k} ; \lambda\right)=\theta^{k}\left[\Gamma(k+1)+\frac{(-1)^{k}-1}{2} \sum_{i=0}^{\infty}(-1)^{i} \frac{(\lambda \theta)^{\alpha i}}{i !} \Gamma(k+\alpha i+1)\right], \quad \lambda>0 .
$$

The characteristic function of the skew Laplace-double Weibull distribution has the form $\phi_{Z}(t)=(1-i t \theta)^{-1}-\frac{1}{2 \theta} \sum_{j=0}^{\infty} \frac{(-1)^{j}}{j !} \lambda^{j \alpha} \Gamma(j \alpha+1)\left[\left(\frac{1}{\theta}-i t\right)^{-(j \alpha+1)}-\left(\frac{1}{\theta}+i t\right)^{-(j \alpha+1)}\right]$.

For $\alpha=2$, we get the skew Laplace-double Raleigh distribution, and, using formula 3.462(5) in Gradshteyn and Ryzhik (1965), the mean of the distribution comes out to be

$$
\mathrm{E}(Z ; \lambda)=\theta-\frac{1}{2 \lambda^{2} \theta}\left[1-\frac{1}{2 \theta} \sqrt{\frac{\pi}{\lambda^{2}}} \exp \left(\frac{1}{4 \lambda^{2} \theta^{2}}\right)\left(1-\Phi\left(\frac{1}{2 \lambda \theta}\right)\right)\right],
$$

where $\Phi(\cdot)$ is the cdf of a standard normal variate.

\section{Skew Laplace-Reflected Gamma Model}

The reflected gamma random variable has the cdf

$$
G(x)=\frac{1}{2}\left[1+\operatorname{sgn}(x) \frac{1}{\Gamma(\alpha)} \gamma(\alpha,|x| / \beta)\right], \quad x \in \mathbb{R}, \quad \alpha, \beta>0,
$$


where $\gamma(a, t)=\int_{0}^{t} x^{\alpha-1} \exp (-x) d x$.

The skew Laplace-reflected gamma distribution then has the pdf

$$
f_{Z}(z ; \lambda)=\frac{1}{2 \theta} \exp (-|z| / \theta)\left[1+\operatorname{sgn}(\lambda z) \frac{1}{\Gamma(\alpha)} \gamma(\alpha,|\lambda z| / \beta)\right], \quad z \in \mathbb{R}, \quad \lambda \in \mathbb{R} .
$$

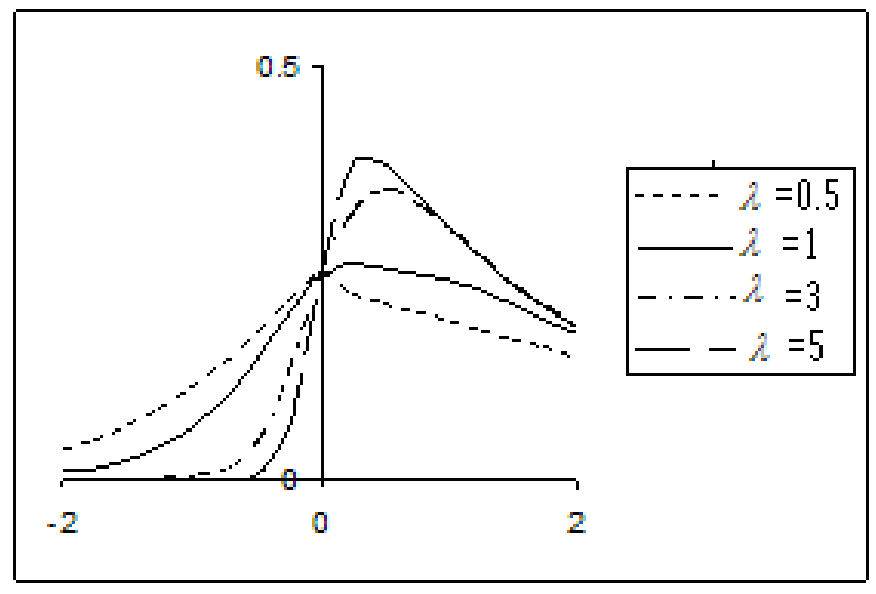

Figure 4: Density curves of skew Laplace-reflected gamma distributions for $\lambda=$ $0.5,1,3,5$ when $\theta=2, \alpha=2, \beta=0.5$.

Using formulas 3.381(4) and 6.455(2) in Gradshteyn and Ryzhik (1965), the $k$-th moment of the distribution is obtained in the following form:

$$
\begin{aligned}
\mathrm{E}\left(Z^{k} ; \lambda\right)= & \theta^{k}\left[\frac{1+(-1)^{k}}{2} k !+\frac{1-(-1)^{k}}{2} \frac{\Gamma(k+\alpha+1)}{\Gamma(\alpha+1)} \frac{\theta^{\alpha}}{(1+\lambda \theta / \beta)^{k+\alpha+1}}\right. \\
& \left.\cdot F\left(1, k+\alpha+1 ; \alpha+1 ; \frac{\lambda \theta / \beta}{1+\lambda \theta / \beta}\right)\right], \quad \lambda>0,
\end{aligned}
$$

where

$$
F(a, b ; c ; x)=\sum_{i=0}^{\infty} \frac{(a)_{i}(b)_{i}}{(c)_{i}} \frac{x^{i}}{i !}
$$

is the Gauss hypergeometric function with Pochhammer symbol

$$
(a)_{i}=\frac{\Gamma(a+i)}{\Gamma(a)}=a(a+1)(a+2) \cdots(a+i-1) .
$$

For $k=1$, using formula 12.2.25 in Abramowitz and Stegun (1972), we get

$$
F(1, \alpha+2 ; \alpha+1 ; x)=\frac{(1+x)(1+\alpha-\alpha x)}{(1+\alpha)(1-x)}
$$

so that

$$
\mathrm{E}(Z ; \lambda)=\theta^{\alpha+1} \frac{(1+2 \lambda \theta / \beta)(1+\alpha+\lambda \theta / \beta)}{(1+\lambda \theta / \beta)^{\alpha+3}} .
$$


The characteristic function of the skew Laplace-reflected gamma distribution is given by

$$
\begin{aligned}
\phi_{Z}(t)=(1-i t \theta)^{-1}- & {\left[\frac{i t \theta}{\left(1+t^{2} \theta^{2}\right)}+\frac{1}{2(1+i t \theta)\left[1+\frac{\beta}{\lambda}\left(\frac{1}{\theta}+i t\right)\right]^{\alpha}}\right.} \\
& \left.-\frac{1}{2(1-i t \theta)\left[1+\frac{\beta}{\lambda}\left(\frac{1}{\theta}-i t\right)\right]^{\alpha}}\right] .
\end{aligned}
$$

\section{Skew Laplace-Reflected Pareto Model}

The reflected Pareto distribution is defined by the cdf

$$
G(x)=\frac{1}{2}\left[1+\operatorname{sgn}(x)\left(1-\left(\frac{\beta}{\beta+|x|}\right)^{\alpha}\right)\right], \quad x \in \mathbb{R}, \quad \alpha, \beta>0 .
$$

A skew Laplace-reflected Pareto distribution, therefore, has the pdf

$$
f_{Z}(z ; \lambda)=\frac{1}{2 \theta} \exp (-|z| / \theta)\left[1+\operatorname{sgn}(\lambda z)\left(1-\left(\frac{\beta}{\beta+|\lambda z|}\right)^{\alpha}\right)\right], \quad z, \lambda \in \mathbb{R} .
$$

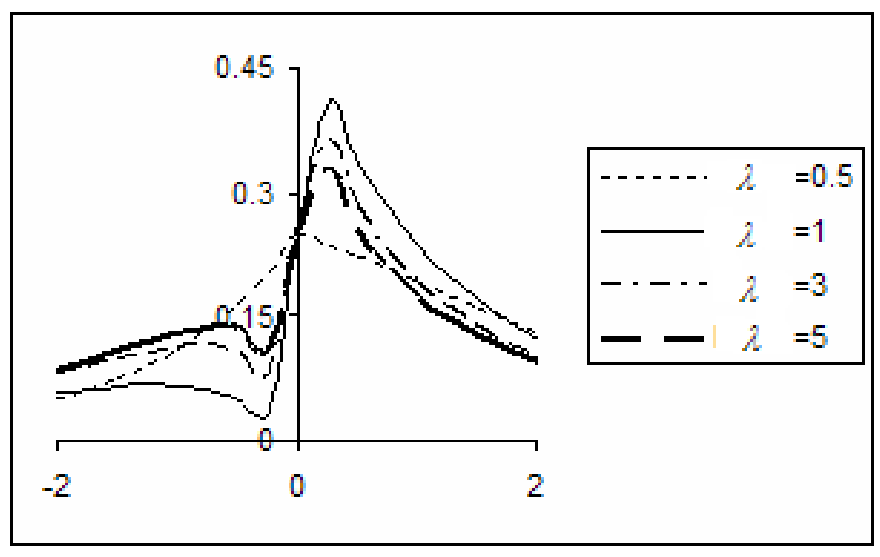

Figure 5: Density curves of skew Laplace-reflected Pareto distributions for $\lambda=0.5,1,3,5$ when $\theta=2, \alpha=2, \beta=3$.

For $\lambda>0$, the $k$-th moment of the distribution is obtained using formula 3.5 in Oberhettinger (1974) in the following form:

$$
\mathrm{E}\left(Z^{k} ; \lambda\right)=\theta^{k} \Gamma(k+1)\left[1+\frac{1-(-1)^{k}}{2} \exp \left(\frac{\beta}{2 \theta \lambda}\right)\left(\frac{\beta}{\theta \lambda}\right)^{\frac{k+\alpha}{2}} W_{-\frac{k+\alpha}{2}, \frac{k-\alpha+1}{2}}\left(\frac{\beta}{\theta \lambda}\right)\right],
$$

where $W_{a, b}(x)$ is the Whittaker function. 
Using formula 3.381(3) in Gradshteyn and Ryzhik (1965) the moment may also be expressed in the following form :

$$
\begin{array}{r}
\mathrm{E}\left(Z^{k} ; \lambda\right)=\theta^{k}\left[\Gamma(k+1)+\frac{(-1)^{k}-1}{2} \exp \left(\frac{\beta}{\theta \lambda}\right) \sum_{i=0}^{k}(-1)^{k-i}\left(\begin{array}{c}
k \\
i
\end{array}\right)\left(\frac{\beta}{\theta \lambda}\right)^{k+\alpha-i}\right. \\
\left.\Gamma\left(i-\alpha+1, \frac{\beta}{\theta \lambda}\right)\right],
\end{array}
$$

where $\Gamma(a, x)=\frac{\gamma(a, x)}{\Gamma(a)}$ is an incomplete gamma function.

The mean of the skewed distribution, therefore, comes out to be

$$
\mathrm{E}(Z ; \lambda)=\theta\left[1+\exp \left(\frac{\beta}{\theta \lambda}\right)\left(\frac{\beta}{\theta \lambda}\right)^{\alpha}\left(\frac{\beta}{\theta \lambda} \Gamma\left(1-\alpha, \frac{\beta}{\theta \lambda}\right)-\Gamma\left(2-\alpha, \frac{\beta}{\theta \lambda}\right)\right)\right] .
$$

Using formula 3.383(5) in Gradshteyn and Ryzhik (1965), the characteristic function of the distribution is given by

$\phi_{Z}(t)=(1-i t \theta)^{-1}-\frac{\beta^{\alpha}}{\theta} \sum_{j=0}^{\infty} \frac{(i t)^{2 j+1}}{j !}\left(\frac{\lambda}{\beta}\right)^{-2(j+1)} \Gamma(2 j+2) \psi\left(2(j+1), 2 j+3-\alpha ; \frac{\beta}{\theta \lambda}\right)$,

where $\psi(a, b ; x)$ is the degenerate hypergeometric function.

\section{Skew Laplace-Reflected Beta Prime Model}

The reflected beta prime distribution has the cdf

$$
G(x)=\frac{1}{2}\left[1+\operatorname{sgn}(x) I_{\frac{|x|}{1+|x|}}(\alpha, \beta)\right], \quad x \in \mathbb{R}, \quad \alpha, \beta>0,
$$

where $I_{x}(a, b)=B_{x}(a, b) / B(a, b)$ is the incomplete beta function.

The pdf of the skew Laplace-beta prime distribution is, therefore,

$$
f_{Z}(z ; \lambda)=\frac{1}{2 \theta} \exp (-|z| / \theta)\left[1+\operatorname{sgn}(\lambda z) I_{\frac{|\lambda z|}{1+|\lambda z|}}(\alpha, \beta)\right], \quad z \in \mathbb{R}, \quad \lambda \in \mathbb{R} .
$$

To find the moments of the distribution we make use of the formulas 3.381(4), 8.391 and 8.392 in Gradshteyn and Ryzhik (1965) and formula 3.5 in Oberhettinger (1974), and get

$$
\begin{aligned}
\mathrm{E}\left(Z^{k} ; \lambda\right)= & \theta^{k}\left[\frac{1+(-1)^{k}}{2} \Gamma(k+1)+\frac{1-(-1)^{k}}{2} \frac{(\theta \lambda)^{-k / 2}}{\alpha B(\alpha, \beta)}\right. \\
& \left.\cdot \exp \left(\frac{1}{2 \theta \lambda}\right) \sum_{j=0}^{\infty} \frac{(\alpha)_{j}(1-\beta)_{j}}{j !(\alpha+1)_{j}} \Gamma(k+\alpha+j+1) W_{-(\alpha+j+k / 2), \frac{k+1}{2}}\left(\frac{1}{\lambda \theta}\right)\right], \\
& \lambda>0 .
\end{aligned}
$$




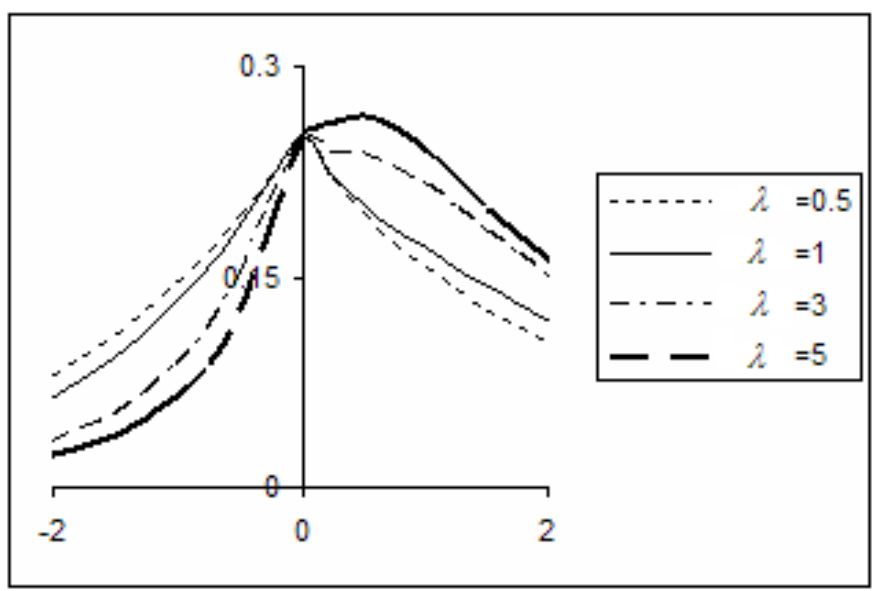

Figure 6: Density curve of skew Laplace-reflected beta prime distribution for $\lambda=$ $0.5,1,3,5$ when $\theta=2, \alpha=2, \beta=1$.

The characteristic function of the skew Laplace-reflected beta prime distribution is obtained as follows, using formula 3.383(5) in Gradshteyn and Ryzhik (1965):

$$
\begin{aligned}
\phi_{Z}(t)=(1-i t \theta)^{-1}- & {\left[\frac{i t \theta}{\left(1+t^{2} \theta^{2}\right)}\right.} \\
+ & \left.\frac{1}{\theta} \sum_{j=0}^{\infty} \frac{(i t / \lambda)^{2 j+1}}{j !} \Gamma(2 j+\alpha+1) \psi\left(2 j+\alpha+1,2 j-\beta+2 ; \frac{1}{\theta \lambda}\right)\right] .
\end{aligned}
$$

\section{Skew Laplace-Reflected Generalized Uniform Model}

A reflected generalized uniform variable $X$ has the cdf given by

$$
G(x)= \begin{cases}0 & \text { if } x<-\beta \\ \frac{1}{2}\left[1+\operatorname{sgn}(x)(|x| / \beta)^{\alpha+1}\right] & \text { if }|x| \leq \beta \\ 1 & \text { if } x>\beta\end{cases}
$$

where $\beta>0, \alpha>-1$. For $\alpha=0$, it becomes the uniform distribution, and for $\beta=1$ we get the reflected power function distribution.

The skew Laplace-reflected generalized uniform distribution will, therefore, be defined by the pdf

$$
f_{Z}(z ; \lambda)= \begin{cases}0 & \text { if } \lambda z<-\beta \\ \frac{1}{2 \theta} \exp (-|z| / \theta)\left[1+\operatorname{sgn}(\lambda z)(|\lambda z| / \beta)^{\alpha+1}\right] & \text { if }|\lambda z| \leq \beta \\ \frac{1}{\theta} \exp (-|z| / \theta) & \text { if } \lambda z>\beta\end{cases}
$$

For $\lambda>0$, we use formulas 3.381(1) and 3.381(3) in Gradshteyn and Ryzhik (1965) 


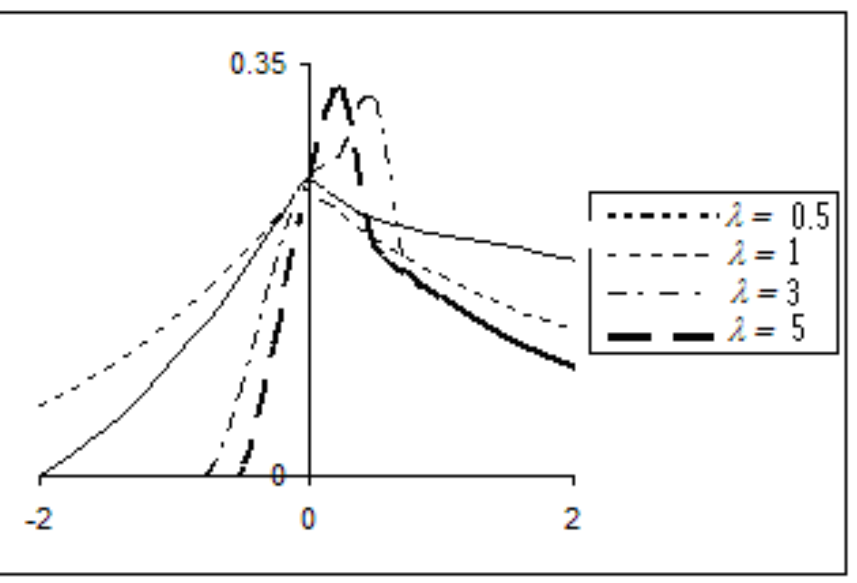

Figure 7: Density curves of skew Laplace-reflected generalized uniform distributions for $\lambda=0.5,1,3,5$ when $\theta=2, \alpha=0.5, \beta=2$.

to get

$$
\begin{aligned}
\mathrm{E}\left(Z^{k} ; \lambda\right)=\theta^{k}[ & \Gamma\left(k+1, \frac{\beta}{\lambda \theta}\right)+\frac{1+(-1)^{k}}{2} \theta \gamma\left(k+1, \frac{\beta}{\lambda \theta}\right) \\
& \left.+\frac{1-(-1)^{k}}{2}\left(\frac{\theta \lambda}{\beta}\right)^{\alpha+1} \gamma\left(k+\alpha+2, \frac{\beta}{\lambda \theta}\right)\right],
\end{aligned}
$$

for $k=1,2, \ldots$ Then,

$$
\mathrm{E}(Z ; \lambda)=\theta\left[\Gamma\left(2, \frac{\beta}{\lambda \theta}\right)+\left(\frac{\theta \lambda}{\beta}\right)^{\alpha+1} \gamma\left(\alpha+3, \frac{\beta}{\lambda \theta}\right)\right], \quad \mathrm{E}\left(Z^{2} ; \lambda\right)=2 \theta^{2} .
$$

For $\alpha=0$, i.e. for the skew Laplace-uniform distribution,

$$
\begin{array}{r}
\mathrm{E}\left(Z^{k} ; \lambda\right)=\theta^{k}\left[\Gamma\left(k+1, \frac{\beta}{\lambda \theta}\right)+\frac{1+(-1)^{k}}{2} \theta \gamma\left(k+1, \frac{\beta}{\lambda \theta}\right)\right. \\
\left.+\frac{1-(-1)^{k}}{2}\left(\frac{\theta \lambda}{\beta}\right) \gamma\left(k+2, \frac{\beta}{\lambda \theta}\right)\right],
\end{array}
$$

for $k=1,2, \ldots$ Hence, for the skew Laplace-uniform distribution,

$$
\mathrm{E}(Z ; \lambda)=\theta\left[\Gamma\left(2, \frac{\beta}{\lambda \theta}\right)+\left(\frac{\theta \lambda}{\beta}\right) \gamma\left(3, \frac{\beta}{\lambda \theta}\right)\right], \quad \mathrm{E}\left(Z^{2} ; \lambda\right)=2 \theta^{2} .
$$

The characteristic function of the skew Laplace-reflected generalized uniform distribution is obtained as

$$
\begin{array}{r}
\phi_{Z}(t)=(1-i t \theta)^{-1}-\frac{i t \theta}{1+t^{2} \theta^{2}}-\frac{1}{2} \exp (-\beta / \theta)\left(\frac{\exp (-i t \beta)}{1+i t \theta}-\frac{\exp (i t \beta)}{1-i t \theta}\right) \\
+(\lambda \theta / \beta)^{\alpha+1} \sum_{j=0}^{\infty} \frac{(i t \theta)^{2 j+1}}{(2 j+1) !} \gamma(2 j+\alpha+3, \beta / \theta) .
\end{array}
$$




\section{Skewness and Kurtosis of the Skewed Distributions}

For the standard Laplace distribution, $\gamma_{1}=0$ and $\gamma_{2}=6$, which means it is a symmetric, platykurtic distribution. In order to examine how flexible the six skewed distributions defined are, in the sense of skewness and peakedness, we draw the skewness-kurtosis graphs (see Dudewicz and Mishra, 1988). The graphs have been drawn by computing $\left(\gamma_{1}, \gamma_{2}\right)$ for the set of parameter values as follows:

- $\theta=1,2, \ldots, 40$ and $\lambda=-100000,-99999, \ldots, 99999,100000$

- $\beta=1,2, \ldots, 40$ for skew Laplace-Laplace distribution

- $\alpha=1,2, \ldots, 40$ for skew Laplace-Double Weibull distribution

- $\beta=1$ and $\alpha=1,2, \ldots, 40$ for skew Laplace-reflected Gamma distribution

- $\alpha=2$ and $\beta=1,2, \ldots, 40$ for skew Laplace-reflected Pareto distribution

- $\alpha=1$ and $\beta=1,2, \ldots, 40$ for skew Laplace-reflected beta prime distribution

- $\alpha=0$ and $\beta=1,2, \ldots, 40$ for skew Laplace-generalized uniform distribution

For the last four distributions, one of the parameters is maintained at a fixed value for the sake of simplicity.

It is quite evident from the graphs that the skewed distributions investigated are very flexible in terms of exhibiting both positive and negative skewness, as well as high and low degrees of peakedness. If variation in values of the third parameter in skew Laplacereflected Gamma, skew Laplace-reflected Pareto, skew Laplace-beta prime distribution and skew Laplace-uniform models be allowed, then wider coverage areas could be realized in the graphs.

\section{Concluding Remarks}

In recent years, a fair amount of research has focused on extending families of symmetric densities to incorporate skewed distributions. This has resulted in a vast literature on skewed distributions, giving a broader flexibility to statistical modeling of natural phenomena. The skewed distributions discussed in this paper add a great deal of flexibility in the sense that by proper choice of skewing parameter, they can be made to exhibit both positive and negative skewness, as well as higher and lower degrees of peakedness.

\section{Acknowledgments}

The authors thank the anonymous referee for several fruitful suggestions, which immensely helped to improve the presentation of the paper.

\section{References}

Abramowitz, M., and Stegun, I. A. (1972). Handbook of mathematical functions with formulas, graphs, and mathematical tables. New York: Dover. 
(a)

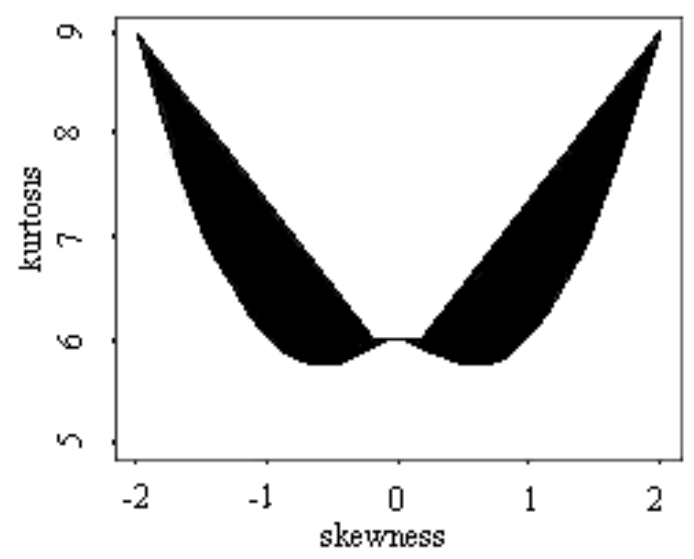

(c)

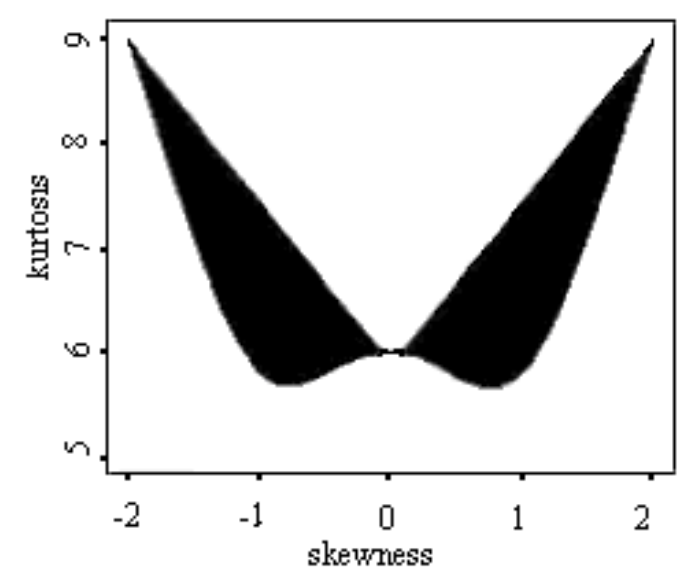

(e)

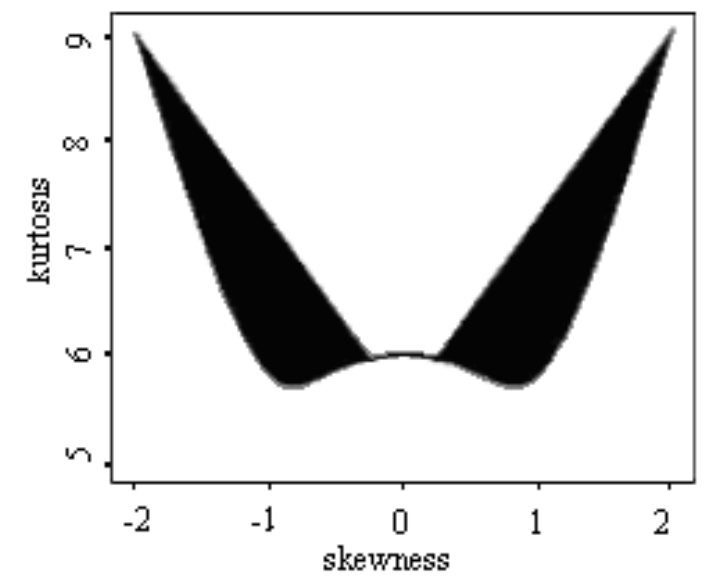

(b)

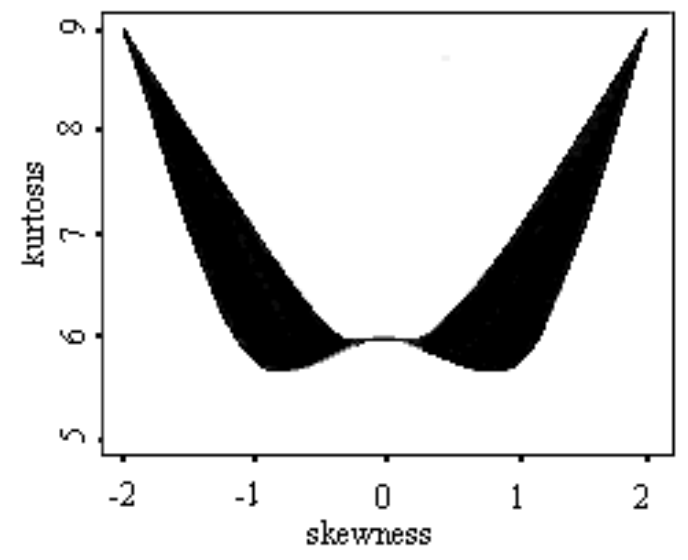

(d)

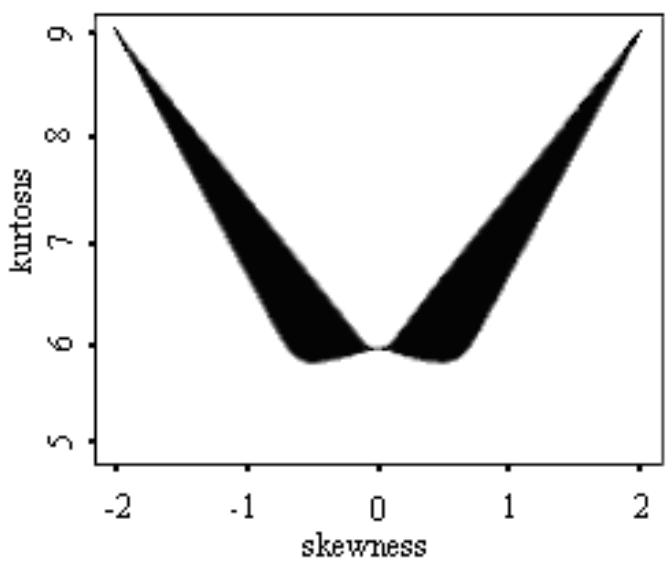

(f)

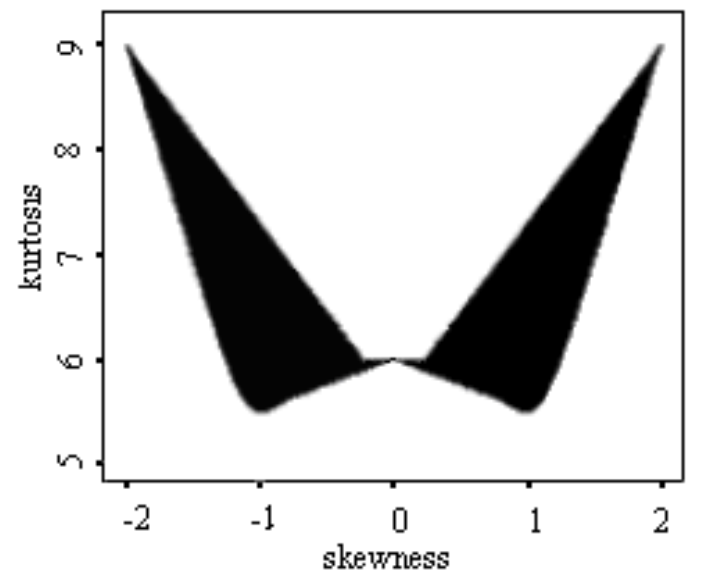

Figure 8: Skewness-kurtosis graphs for (a) skew Laplace-Laplace distribution, (b) skew Laplace-double Weibull distribution, (c) skew Laplace-reflected gamma distribution, (d) skew Laplace-reflected Pareto distribution, (e) skew Laplace-beta prime distribution, and (f) skew Laplace-uniform distribution. 
Ali, M. M., Pal, M., and Woo, J. (2008). Skewed reflected distributions generated by reflected gamma kernel. Pakistan Journal of Statistics, 24, 77-86.

Ali, M. M., and Woo, J. (2006). Skew-symmetric reflected distributions. Soochow Journal of Mathematics, 32, 233-240.

Ali, M. M., Woo, J., Pal, M., and Wahed, A. S. (2008). Some skew-symmetric double inverted distributions. International Journal of Statistical Sciences, 7, 1-12.

Arnold, B. C., and Beaver, R. J. (2000a). Some skewed multivariate distributions. American Journal of Mathematical and Management Sciences, 20, 27-38.

Arnold, B. C., and Beaver, R. J. (2000b). The skew-Cauchy distribution. Statistics and Probability Lettters, 49, 285-290.

Arnold, B. C., Beaver, R. J., Groeneveld, R. A., and Meeker, W. Q. (1983). The nontruncated marginal of a truncated bivariate normal distribution. Psychometrika, 58, 471-488.

Azzalini, A. (1985). A class of distributions which includes the normal ones. Scandinavian Journal of Statistics, 12, 171-178.

Azzalini, A. (1986). Further results on a class of distributions which includes the normal ones. Statistica, 46, 199-208.

Azzalini, A., and Capitanio, A. (1999). Statistical applications of the multivariate skewnormal distribution. Journal of the Royal Statistical Society, Series B, 61, 579-602.

Azzalini, A., and Dalla Valle, A. (1996). The multivariate skewed normal distribution. Biometrika, 83, 715-726.

Balakrishnan, N., and Ambagaspitiy, R. S. (1994). On skew-Laplace distributions (Tech. Rep.). Department of Mathematics and Statistics, McMaster University, Hamilton, Ontario, Canada.

Dudewicz, E. J., and Mishra, S. N. (1988). Modern Mathematical Statistics. New York: John Wiley and Sons, Inc.

Gradshteyn, I. S., and Ryzhik, I. M. (1965). Tables of Integrals, Series and Products. New York: Academic Press.

Gupta, A. K., Chang, F. C., and Huang, W. J. (2002). Some skew-symmetric models. Random Operators and Stochastic Equations, 10, 133-140.

Hill, M. A., and Dixon, W. J. (1982). Robustness in real life: a study of clinical laboratory data. Biometrics, 38, 377-396.

Mukhopadhyay, S., and Vidakovic, B. (1995). Efficiency of linear bayes rules for a normal mean: skewed priors class. The Statistician, 44, 389-397.

Nadarajah, S., and Kotz, S. (2003). Skewed distributions generated by the normal kernel. Statistics and Probability Lettters, 65, 269-277.

Nadarajah, S., and Kotz, S. (2004). Skewed distribution generated by the Laplace kernel. American Journal of Mathematical and Management Sciences, 24, 321-349.

Oberhettinger, F. (1974). Tables of Mellin Transforms. New York: Springer-Verlag.

O'Hagan, A., and Leonard, T. (1976). Bayes estimation subject to uncertainty about parameter constraints. Biometrika, 63, 201-203.

Wahed, A. S., and Ali, M. M. (2001). The skewed logistic distribution. Journal of Statistical Research, 35, 71-80. 
Corresponding Author's Address:

Manisha Pal

Department of Statistics

University of Calcutta

35 Ballygunge Circular Road

Kolkata 700019

India

E-mail: manishapal2@gmail.com 